\title{
CHAIN CONDITIONS FOR LEAVITT PATH ALGEBRAS
}

\author{
G. ABRAMS, G. ARANDA PINO, F. PERERA, AND M. SILES MOLINA
}

\begin{abstract}
In this paper, results known about the artinian and noetherian conditions for the Leavitt path algebras of graphs with finitely many vertices are extended to all row-finite graphs. In our first main result, necessary and sufficient conditions on a row-finite graph $E$ are given so that the corresponding (not necessarily unital) Leavitt path $K$-algebra $L(E)$ is semisimple. These are precisely the algebras $L(E)$ for which every corner is left (equivalently, right) artinian. They are also precisely the algebras $L(E)$ for which every finitely generated left (equivalently, right) $L(E)$-module is artinian. In our second main result, we give necessary and sufficient conditions for every corner of $L(E)$ to be left (equivalently, right) noetherian. They also turn out to be precisely those algebras $L(E)$ for which every finitely generated left (equivalently, right) $L(E)$-module is noetherian. In both situations, isomorphisms between these algebras and appropriate direct sums of matrix rings over $K$ or $K\left[x, x^{-1}\right]$ are provided. Likewise, in both situations, equivalent graph theoretic conditions on $E$ are presented.
\end{abstract}

\section{INTRODUCTION}

Leavitt path algebras $L_{K}(E)$ of row-finite graphs have been recently introduced in [1] and [7]. They have become a subject of significant interest, both for algebraists and for analysts working in $\mathrm{C}^{*}$-algebras. The CuntzKrieger algebras $C^{*}(E)$ (the $\mathrm{C}^{*}$-algebra counterpart of these Leavitt path algebras) are described in [19]. The algebraic and analytic theories share some striking similarities, as well as some distinct differences, as has been shown for instance in the "Workshop on Graph Algebras" held at the University of Málaga (see [10]), and more deeply in the subsequent enlightening work of Tomforde [21].

For a field $K$, the algebras $L_{K}(E)$ are natural generalizations of the algebras investigated by Leavitt in [18], and are a specific type of path $K$ algebras associated to a graph $E$ (modulo certain relations). The family of algebras which can be realized as the Leavitt path algebras of a graph

2000 Mathematics Subject Classification. Primary 16D70.

Key words and phrases. Leavitt path algebra, semisimple, locally artinian, locally noetherian. 
includes matrix rings $\mathbb{M}_{n}(K)$ for $n \in \mathbb{N} \cup\{\infty\}$ (where $\mathbb{M}_{\infty}(K)$ denotes matrices of countable size with only a finite number or nonzero entries), the Toeplitz algebra, the Laurent polynomial ring $K\left[x, x^{-1}\right]$, and the classical Leavitt algebras $L(1, n)$ for $n \geq 2$. Constructions such as direct sums, direct limits, and matrices over the previous examples can be also realized in this setting.

The literature on Leavitt path algebras includes necessary and sufficient conditions on a graph $E$ so that the corresponding Leavitt path algebra is simple [1], purely infinite simple [2], exchange [9], finite-dimensional [4], and locally finite (equivalently, noetherian) [5]. The study of the monoids of finitely generated projective modules over $L_{K}(E)$ was done in [7], while in [20] it was shown that the Leavitt path algebra $L_{K}(E)$ is an algebra of right quotients of the path algebra $K E$. Very recently too, the structure of the socle of the Leavitt path algebra was studied in [8]. Knowledge of the structure of the socle turns out to be a key ingredient in the study of the rings under consideration in this paper.

The article is organized as follows. The Preliminaries section includes the basic definitions and examples that will be used throughout. In addition, we describe categorically artinian (resp. categorically noetherian) rings; these are (possibly nonunital) rings for which every finitely generated module is artinian (resp. noetherian).

In Section 2 the study of semisimple Leavitt path algebras is carried out. We start by establishing the equivalence between finite-dimensional Leavitt path algebras and unital left (equivalently, right) artinian Leavitt path algebras. Consistent with the approach taken throughout this article, we then use this information together with previously known results to give equivalent categorical, ring-theoretic, graph-theoretic, and explicit descriptions of the finite-dimensional Leavitt path algebras. One such description is that the finite-dimensional Leavitt path algebras are precisely the unital semisimple Leavitt path algebras. Further along in Section 2, the characterization of all (i.e, not necessarily unital) semisimple Leavitt path algebras is obtained (Theorem 2.6). These are the locally left (equivalently, right) artinian Leavitt path algebras, that is, the class of rings $R$ such that every corner $e R e$ for $e$ an idempotent in $R$ is left (equivalently, right) artinian. Indeed, these are precisely the categorically left (equivalently, right) artinian Leavitt path algebras. Furthermore, these rings comprise precisely the class of von Neumann regular Leavitt path algebras such that the monoid $V\left(L_{K}(E)\right)$ of finitely generated projective left $L_{K}(E)$-modules is cancellative (in fact, isomorphic to a finite or countable product of copies of $\mathbb{Z}^{+}$). Analogous to what was done for the unital artinian Leavitt path algebras, we specify equivalent categorical, ring-theoretic, graph-theoretic, and 
explicit descriptions of the semisimple Leavitt path algebras. Informally, they are the (perhaps infinite) direct sum of (possibly countably sized) matrix rings over the field $K$, and they arise from the acyclic graphs $E$ having the property that every infinite path ends in a sink.

The natural subsequent step is taken in Section 3, where we study the noetherian condition on Leavitt path algebras. The unital noetherian Leavitt path algebras were classified in [5]. The extension of the noetherian condition to graphs with infinitely many vertices proceeds in a manner much akin to the artinian situation. Specifically, we show that the class of locally noetherian Leavitt path algebras (i.e., rings in which every corner is noetherian) is precisely the class of categorically noetherian rings. Furthermore, again analogous to previous results, we provide equivalent categorical, ring-theoretic, graph-theoretic, and explicit descriptions for this type of algebras. Informally, they are the (perhaps infinite) direct sum of (possibly countably sized) matrix rings over the field $K$ or over the algebra of Laurent polynomials $K\left[x, x^{-1}\right]$, and they arise from the graphs $E$ satisfying both Condition (NE) (i.e., No cycle has an Exit), and the condition that every infinite path ends either in a sink or in a cycle.

\section{Preliminaries}

A (directed) graph $E=\left(E^{0}, E^{1}, r, s\right)$ consists of two countable sets $E^{0}, E^{1}$ and maps $r, s: E^{1} \rightarrow E^{0}$. The elements of $E^{0}$ are called vertices and the elements of $E^{1}$ edges. If $s^{-1}(v)$ is a finite set for every $v \in E^{0}$, then the graph is called row-finite. Throughout this paper we will be concerned only with row-finite graphs. If $E^{0}$ is finite, then, by the row-finite hypothesis, $E^{1}$ must necessarily be finite as well; in this case we say simply that $E$ is finite. A vertex which emits no edges is called a sink. A path $\mu$ in a graph $E$ is a sequence of edges $\mu=e_{1} \ldots e_{n}$ such that $r\left(e_{i}\right)=s\left(e_{i+1}\right)$ for $i=1, \ldots, n-1$. In this case, $s(\mu):=s\left(e_{1}\right)$ is the source of $\mu, r(\mu):=r\left(e_{n}\right)$ is the range of $\mu$, and $n$ is the length of $\mu$. For $n \geq 2$ we define $E^{n}$ to be the set of paths of length $n$, and $E^{*}=\bigcup_{n \geq 0} E^{n}$ the set of all paths. Throughout the paper $K$ will denote an arbitrary field.

We define the Leavitt path $K$-algebra $L_{K}(E)$, or simply $L(E)$ if the base field is understood, as the $K$-algebra generated by a set $\left\{v \mid v \in E^{0}\right\}$ of pairwise orthogonal idempotents, together with a set of variables $\left\{e, e^{*} \mid\right.$ $\left.e \in E^{1}\right\}$, which satisfy the following relations:

(1) $s(e) e=e r(e)=e$ for all $e \in E^{1}$.

(2) $r(e) e^{*}=e^{*} s(e)=e^{*}$ for all $e \in E^{1}$.

(3) $e^{*} e^{\prime}=\delta_{e, e^{\prime}} r(e)$ for all $e, e^{\prime} \in E^{1}$.

(4) $v=\sum_{\left\{e \in E^{1} \mid s(e)=v\right\}} e e^{*}$ for every $v \in E^{0}$ that emits edges. 
The elements of $E^{1}$ are called real edges, while for $e \in E^{1}$ we call $e^{*}$ a ghost edge. The set $\left\{e^{*} \mid e \in E^{1}\right\}$ will be denoted by $\left(E^{1}\right)^{*}$. We let $r\left(e^{*}\right)$ denote $s(e)$, and we let $s\left(e^{*}\right)$ denote $r(e)$. If $\mu=e_{1} \ldots e_{n}$ is a path, then we denote by $\mu^{*}$ the element $e_{n}^{*} \ldots e_{1}^{*}$ of $L(E)$, and by $\mu^{0}$ the set of its vertices, i.e., $\left\{s\left(\mu_{1}\right), r\left(\mu_{i}\right) \mid i=1, \ldots, n\right\}$. It was shown in [1, Lemma 1.5] that every monomial in $L(E)$ is of the form: $k v$, with $k \in K$ and $v \in E^{0}$, or $k e_{1} \ldots e_{m} f_{1}^{*} \ldots f_{n}^{*}$ for $k \in K, m, n \in \mathbb{N}, e_{i}, f_{j} \in E^{1}$. For any subset $H$ of $E^{0}$, we will denote by $I(H)$ the ideal of $L(E)$ generated by $H$.

Note that if $E$ is a finite graph then we have $\sum_{v \in E^{0}} v=1_{L(E)}$. On the other hand, if $E^{0}$ is infinite, then by [1, Lemma 1.6] $L(E)$ is a nonunital ring with a set of local units. In fact, in this situation, $L(E)$ is a ring with enough idempotents (see e.g. [15] or [21]), and we have the decomposition $L(E)=\oplus_{v \in E^{0}} L(E) v$ as left $L(E)$-modules. (Equivalently, we have $L(E)=\oplus_{v \in E^{0}} v L(E)$ as right $L(E)$-modules.) If $R$ is a ring with enough idempotents, then by a left $R$-module we mean a unitary left $R$-module, that is, a module $M$ in the usual sense with the added condition that $R M=M$.

Definition 1.1. Let $R$ be a ring with local units.

(i) We say $R$ is categorically left artinian in case every finitely generated left $R$-module is artinian.

(ii) We say $R$ is categorically left noetherian in case every finitely generated left $R$-module is noetherian.

The analogous definitions of categorically right artinian and categorically right noetherian are obvious. These concepts will allow us to extend appropriately the corresponding notions from the unital case, as is evident from the following proposition.

Proposition 1.2. Suppose $E$ is a set of idempotents in the ring $R$ for which $R=\oplus_{e \in E} R e$. Then $R$ is categorically left artinian (resp. noetherian) if and only if each Re is a left artinian (resp. noetherian) $R$-module. In particular, if $R$ is a unital ring, then $R$ is left artinian (resp. noetherian) if and only if $R$ is categorically left artinian (resp. noetherian).

Proof. The left regular module $R$ is a generator for the category $R-M o d$ of left $R$-modules. (This is of course true for unital rings, but in fact, by the hypothesis that $R M=M$ for each left $R$-module $M$, follows easily for rings with enough idempotents as well.) If each $R e$ is artinian (resp. noetherian), then standard arguments yield that any factor of any finite direct sum of such modules is also artinian (resp. noetherian), so that any finitely generated left $R$-module is artinian (resp. noetherian) as well. The second statement is then obvious, as $R=R \cdot 1$. 
For us, by a countable set we mean a set which is either finite or countably infinite. The symbol $\mathbb{M}_{\infty}(K)$ will denote the $K$-algebra of matrices over $K$ of countable size but with only a finite number of nonzero entries.

\section{Corollary 1.3.}

(i) Any ring of the form

$$
\bigoplus_{i \in \Upsilon} \mathbb{M}_{n_{i}}(K)
$$

where $\Upsilon$ is a countable set and $n_{i} \in \mathbb{N} \cup\{\infty\}$, is categorically artinian.

(ii) Any ring of the form

$$
\bigoplus_{i \in \Upsilon_{1}} \mathbb{M}_{n_{i}}(K) \oplus \bigoplus_{j \in \Upsilon_{2}} \mathbb{M}_{m_{j}}\left(K\left[x, x^{-1}\right]\right)
$$

where $\Upsilon_{1}$ and $\Upsilon_{2}$ are countable sets and $n_{i}, m_{j} \in \mathbb{N} \cup\{\infty\}$, is categorically noetherian.

Proof. Let $T$ be any unital ring, let $R=\mathbb{M}_{n}(T)$ where $n \in \mathbb{N} \cup\{\infty\}$, and let $e=e_{i i}$ denote any standard matrix idempotent in $R$. It is straightforward to show that there is an inclusion-preserving bijection between the set of left ideals of $T$ and the set of $R$-submodules of $R e$. (More specifically, every $R$-submodule of $\mathbb{M}_{n}(T) e$ is of the form $\mathbb{M}_{n}(I) e$ for $I$ a left ideal of $T$.) As any field $K$ is artinian, statement (i) then follows from this observation and Proposition 1.2. As the Laurent polynomial algebra $K\left[x, x^{-1}\right]$ is noetherian for any field $K$, statement (ii) follows similarly.

We note here that if $R$ is a nonunital ring with enough idempotents, then the decomposition $R=\oplus_{e \in E} R e$ shows that $R$ can never be left artinian (resp. noetherian) in the usual sense. Thus the notion of categorically artinian (resp. noetherian) is the germane one here.

\section{Definition 1.4.}

(i) Following [14], the ring $R$ is called locally left (resp. right) artinian if for any finite subset $S$ of $R$ there exists $e=e^{2} \in R$ such that $S \subseteq e R e$, with $e$ Re left (resp. right) artinian.

(ii) The ring $R$ is called locally left (resp. right) noetherian if for any finite subset $S$ of $R$ there exists $e=e^{2} \in R$ such that $S \subseteq e R e$, with $e R e$ left (resp. right) noetherian.

As it turns out, the locally artinian condition is characterized, in the setting of semiprime rings, by the fact that every local ring at an element is artinian, equivalently, they coincide with their socle. For a ring $R$ and an element $a \in R$, the local ring of $R$ at $a$ (denoted $R_{a}$ ) is defined to be the ring $a R a$, with the sum inherited from $R$, and product given by $a x a \cdot a y a=$ axaya. (See [16] for an equivalent definition and information about the 
exchange of properties between a ring and its local rings at elements.) In particular, if $e$ is an idempotent in the ring $R$, then the local ring of $R$ at $e$ is just the corner $e R e$. We will investigate this further in Section 2 .

By the definition of a set of local units, it is easy to see that a ring $R$ is locally left or right artinian (resp. noetherian) precisely when $R$ has a set of local units $E$ for which $e$ Re is left or right artinian (resp. noetherian) for each $e \in E$.

Clearly if $R$ is unital, then $R$ is locally left (resp. right) artinian if and only if $R$ is left (resp. right) artinian; it was noted above that in this situation $R$ is necessarily categorically artinian as well. However, in the nonunital setting the categorically artinian and locally artinian properties need not be the same. For instance, let $R$ be the ring $T_{\mathbb{N}}(K)$ of countably infinite square matrices which are lower triangular, and have at most finitely many nonzero entries. Then $T$ is locally artinian, since for each matrix idempotent $f$ the algebra $f T f$ is finite dimensional. However, the finitely generated left $T$-module $T e_{11}$ is not left artinian, since it is easy to check that $T e_{11} \supsetneq T e_{21} \supsetneq T e_{31} \supsetneq \ldots$... (A generalization of this example is presented in $[14$, p. 1256].) Nonetheless, the converse implication does hold.

Lemma 1.5. Let $R$ be a ring with local units. If $R$ is categorically left (resp. right) artinian, then $R$ is locally left (resp. right) artinian.

Proof. We do the "left" case, the "right" case is virtually identical. Let $E$ be a set of local units for $R$. It suffices to show that $e R e$ is left artinian for every $e \in E$. Since the left ideal $R e$ is finitely generated, it is by hypothesis artinian. Now consider a decreasing sequence of left $e R e$-ideals $I_{1} \supseteq I_{2} \supseteq \ldots$ . Then $R I_{1} \supseteq R I_{2} \supseteq \ldots$ is a decreasing sequence of $R$-submodules of $R e$, hence the sequence stabilizes, so that $R I_{k}=R I_{k+1}=\ldots$ for some integer $k$, which in turn yields $e R I_{k}=e R I_{k+1}=\ldots$. But for each positive integer $j$ we have $e R I_{j}=I_{j}$ (because $I_{j} \subseteq e R e$ gives $e I_{j}=I_{j}$ ), so that we get $I_{k}=I_{k+1}=\ldots$ and we are done.

We have an analogous result in the noetherian situation. An example of a ring with local units which is locally noetherian but not categorically noetherian is given in $[14$, p. 1256] (take $X$ to be the chain of real numbers, for instance.) But, arguing as in Lemma 1.5, we get

Lemma 1.6. Let $R$ be a ring with local units. If $R$ is categorically left (resp. right) noetherian, then $R$ is locally left (resp. right) noetherian.

We will analyze the structure of various graphs in the sequel. An important role is played by the following three concepts. An edge $e$ is an exit for a path $\mu=e_{1} \ldots e_{n}$ if there exists $i$ such that $s(e)=s\left(e_{i}\right)$ and $e \neq e_{i}$. If $\mu$ 
is a path in $E$, and if $v=s(\mu)=r(\mu)$, then $\mu$ is called a closed path based at $v$. If $s(\mu)=r(\mu)$ and $s\left(e_{i}\right) \neq s\left(e_{j}\right)$ for every $i \neq j$, then $\mu$ is called a cycle. A graph which contains no cycles is called acyclic.

We define a relation $\geq$ on $E^{0}$ by setting $v \geq w$ if there is a path $\mu \in E^{*}$ with $s(\mu)=v$ and $r(\mu)=w$. A subset $H$ of $E^{0}$ is called hereditary if $v \geq w$ and $v \in H$ imply $w \in H$. A hereditary set is saturated if every vertex which feeds into $H$ and only into $H$ is again in $H$, that is, if $s^{-1}(v) \neq \emptyset$ and $r\left(s^{-1}(v)\right) \subseteq H$ imply $v \in H$. Denote by $\mathcal{H}$ (or by $\mathcal{H}_{E}$ when it is necessary to emphasize the dependence on $E$ ) the set of hereditary saturated subsets of $E^{0}$.

The set $T(v)=\left\{w \in E^{0} \mid v \geq w\right\}$ is the tree of $v$, and it is the smallest hereditary subset of $E^{0}$ containing $v$. We extend this definition for an arbitrary set $X \subseteq E^{0}$ by $T(X)=\bigcup_{x \in X} T(x)$. The hereditary saturated closure of a set $X$ is defined as the smallest hereditary and saturated subset of $E^{0}$ containing $X$. It is shown in [7] that the hereditary saturated closure of a set $X$ is $\bar{X}=\bigcup_{n=0}^{\infty} \Lambda_{n}(X)$, where

$$
\begin{aligned}
& \Lambda_{0}(X)=T(X), \text { and } \\
& \Lambda_{n}(X)=\left\{y \in E^{0} \mid s^{-1}(y) \neq \emptyset \text { and } r\left(s^{-1}(y)\right) \subseteq \Lambda_{n-1}(X)\right\} \cup \\
& \Lambda_{n-1}(X), \text { for } n \geq 1
\end{aligned}
$$

Recall that a vertex $v$ in $E^{0}$ is a bifurcation (or that there is a bifurcation at $v)$ if $s^{-1}(v)$ has at least two elements, and we say that there exists a cycle at $v$ if $v$ is a vertex of some cycle. A vertex $u$ in $E^{0}$ will be called a line point if there are neither bifurcations nor cycles at any vertex $w \in T(u)$. We will denote by $P_{l}(E)$ the set of all line points in $E^{0}$. Clearly $P_{l}(E)$ is always a hereditary set.

Definitions 1.7. We say that an infinite path $\gamma=\left(e_{n}\right)_{n=1}^{\infty}$ ends in a sink if there exists $m \geq 1$ such that the infinite subpath $\mu=\left(e_{n}\right)_{n=m}^{\infty}$ has neither bifurcations nor cycles, or equivalently, if $\mu^{0} \subseteq P_{l}(E)$. The infinite path $\mu$ is called an infinite sink.

Definition 1.8. We say that an infinite path $\gamma=\left(e_{n}\right)_{n=1}^{\infty}$ ends in a cycle if there exists $m \geq 1$ and a cycle $c$ such that the infinite subpath $\left(e_{n}\right)_{n=m}^{\infty}$ is just the infinite path $c c c \ldots$.

We recall here some graph-theoretic constructions which will be of interest. For a hereditary subset of $E^{0}$, the quotient graph $E / H$ is defined as

$$
\left(E^{0} \backslash H,\left\{e \in E^{1} \mid r(e) \notin H\right\},\left.r\right|_{(E / H)^{1}},\left.s\right|_{(E / H)^{1}}\right),
$$

and the restriction graph is

$$
E_{H}=\left(H,\left\{e \in E^{1} \mid s(e) \in H\right\},\left.r\right|_{\left(E_{H}\right)^{1}},\left.s\right|_{\left(E_{H}\right)^{1}}\right) .
$$


The definitions of the following graph are particular cases of those appearing in [13, Definition 1.3]:

Let $E$ be a graph, and let $\emptyset \neq H \in \mathcal{H}_{E}$. Define

$$
\begin{gathered}
F_{E}(H)=\left\{\alpha=\left(\alpha_{1}, \ldots, \alpha_{n}\right) \quad \mid \alpha_{i} \in E^{1}, s\left(\alpha_{1}\right) \in E^{0} \backslash H, r\left(\alpha_{i}\right) \in E^{0} \backslash H\right. \\
\text { for } \left.i<n, r\left(\alpha_{n}\right) \in H\right\} .
\end{gathered}
$$

Denote by $\bar{F}_{E}(H)$ another copy of $F_{E}(H)$. For $\alpha \in F_{E}(H)$, we write $\bar{\alpha}$ to denote a copy of $\alpha$ in $\bar{F}_{E}(H)$. Then, we define the graph ${ }_{H} E=$ $\left({ }_{H} E^{0},{ }_{H} E^{1}, s^{\prime}, r^{\prime}\right)$ as follows:

(1) ${ }_{H} E^{0}=\left({ }_{H} E\right)^{0}=H \cup F_{E}(H)$.

(2) ${ }_{H} E^{1}=\left({ }_{H} E\right)^{1}=\left\{e \in E^{1} \mid s(e) \in H\right\} \cup \bar{F}_{E}(H)$.

(3) For every $e \in E^{1}$ with $s(e) \in H, s^{\prime}(e)=s(e)$ and $r^{\prime}(e)=r(e)$.

(4) For every $\bar{\alpha} \in \bar{F}_{E}(H), s^{\prime}(\bar{\alpha})=\alpha$ and $r^{\prime}(\bar{\alpha})=r(\alpha)$.

\section{Artinian Leavitt path algebras}

In this section we focus our attention on algebraic and graph theoretic characterizations of artinian Leavitt path algebras. This study can be regarded as a natural followup of the work done in [4] for finite-dimensional Leavitt path algebras, and the work done in [8], where the socle of a Leavitt path algebra was described.

Our goal in this article is to study chain conditions on the one-sided ideals of Leavitt path algebras. It is well known that for any unital $K$ algebra $R$, a subset $I$ of $R$ is a left (resp. right) ideal of $R$ if and only if $I$ is a left (resp. right) algebra ideal of $R$ (i.e., $I$ is also closed under scalar multiplication by $K$ ). It is not hard to show that this same property holds for any $K$-algebra $R$ which has enough idempotents (see e.g. [21, Lemma 3.11]). Consequently, when we describe chain conditions on Leavitt path algebras, these conditions may be viewed equivalently as chain conditions in either the ring-theoretic or the algebra-theoretic sense.

On a similar note, throughout the article we will have occasion to establish isomorphisms between Leavitt path algebras and various other $K$ algebras (e.g., direct sums of matrix rings over $K$ or over $\left.K\left[x, x^{-1}\right]\right)$. Because our primary interest here will be in the ring-theoretic structure of these Leavitt path algebras, it is sufficient for our needs to establish simply that such isomorphisms are ring isomorphisms. In fact, all the isomorphisms we will establish between these $K$-algebras can be checked to be $K$-algebra isomorphisms as well. Thus the reader may interpret the symbol $\cong$ either as "ring isomorphic to", or as " $K$-algebra isomorphic to", throughout the article. 
A semiprime ring $A$ is called semisimple if it is the sum (equivalently, the direct sum) of its minimal left ideals (equivalently, of its minimal right ideals). This is equivalent to saying that $A$ is semiprime and coincides with its socle. If $A$ is a ring with local units (or, more generally, a ring for which the left regular module ${ }_{A} A$ is a generator for $A-M o d$ ), then this condition is equivalent to saying that every left $A$-module is a direct sum of simple submodules.

For an arbitrary algebra, finite-dimensional implies left (and right) artinian. The following result shows that for Leavitt path algebras the converse holds too.

Proposition 2.1. Let $E$ be a finite graph. The Leavitt path algebra $L(E)$ is left (equivalently, right) artinian if and only if $L(E) \cong \bigoplus_{i=1}^{t} \mathbb{M}_{n_{i}}(K)$ for some positive integers $\left\{n_{1}, \ldots, n_{t}\right\}$.

Proof. The "only if" direction is clear. Conversely, assume that $L(E)$ is left artinian. By the finiteness of $E, L(E)$ is unital. So we may invoke the structure theorem of semiprime artinian rings (see, for example Jacobson's book [17]), specifically, that semiprime and artinian is equivalent to being semisimple and artinian. Since $L(E)$ is semiprime ([8, Proposition 1.1]), it is a direct sum of matrix rings over division rings. On the other hand we know that for every minimal left ideal $I$ of $L(E)$ there exists a vertex $v \in P_{l}(E)$ such that $I \cong L(E) v([8$, Theorem 3.3]) and $v L(E) v \cong K([8$, Proposition 2.7]), therefore $L(E) \cong \bigoplus_{i=1}^{t} \mathbb{M}_{n_{i}}(K)$ as desired. The right artinian case is similar.

Proposition 2.1 provides a key step towards the following important consequence.

Theorem 2.2. Let $E$ be a finite graph (in other words, let $L(E)$ be unital). The following conditions are equivalent.

(i) $L(E)$ is semisimple.

(ii) $L(E)$ is left artinian.

(ii) $^{\prime} L(E)$ is right artinian.

(iii) $L(E) \cong \bigoplus_{i=1}^{t} \mathbb{M}_{n_{i}}(K)$ for some positive integers $\left\{n_{1}, \ldots, n_{t}\right\}$.

(iv) $E$ is acyclic.

(v) $L(E)$ is finite dimensional.

Proof. (v) $\Rightarrow$ (iv) is [9, Proposition 3.6], while (iv) $\Rightarrow$ (iii) is [9, Proposition 3.5]. The implications (iii) $\Rightarrow$ (i), (iii) $\Rightarrow$ (ii), (iii) $\Rightarrow$ (ii) $^{\prime}$, (i) $\Rightarrow$ (ii), and (i) $\Rightarrow\left(\right.$ ii) ${ }^{\prime}$ are well known. But by Proposition 2.1 both (ii) and (ii)' imply (iii), which in turn clearly implies (v), and we are done. 
We now develop the ideas which will allow us to extend Theorem 2.2 to all row-finite graphs.

Lemma 2.3. Let e be an idempotent in a Leavitt path algebra $L(E)$. The corner eL $(E) e$ is a division ring if and only if it is isomorphic to $K$ as a ring.

Proof. Suppose that $e L(E) e$ is a division ring. Recall that the Leavitt path algebra is semiprime by [8, Proposition 1.1]. As such, an application of [17, $\S$ IV.3, Proposition 1] yields that division corners $e L(E) e$ give rise to minimal right (respectively, left) ideals $e L(E)$ (respectively, $L(E) e)$. Thus, $L(E) e$ is a minimal left ideal and by [8, Theorem 3.3], there exists $v \in P_{l}(E)$ such that $L(E) e$ is isomorphic to $L(E) v$ as left $L(E)$-modules. In this situation [8, Proposition 2.7] applies to give that $v L(E) v=K v \cong K$ as rings.

To finish the proof we perform the following isomorphisms: Since $L(E) e \cong$ $L(E) v$ as left $L(E)$-modules, then $E n d_{L(E)}(L(E) e) \cong E n d_{L(E)}(L(E) v)$ as rings. But it is well-known that for any ring $R$ and any idempotent $e \in R$ we have $\operatorname{End}_{R}(R e) \cong e R e$ as rings. Thus, we obtain the following ring isomorphisms

$$
e L(E) e \cong \operatorname{End}_{L(E)}(L(E) e) \cong \operatorname{End}_{L(E)}(L(E) v) \cong v L(E) v \cong K .
$$

The converse is obvious.

Lemma 2.4. Let $E$ be any row-finite graph. The Leavitt path algebra $L(E)$ is semisimple if and only if $\overline{P_{l}(E)}=E^{0}$.

Proof. We know from [8, Theorem 4.2] that $\operatorname{Soc}(L(E))=I\left(\overline{P_{l}(E)}\right)$, so that $L(E)$ is semisimple if and only if $L(E)=I\left(\overline{P_{l}(E)}\right)$. Now use the lattice isomorphism between graded ideals $\mathcal{L}_{g r}(L(E))$ and the hereditary saturated subsets $\mathcal{H}$ explained in $\left[9\right.$, Remark 2.2] to get that $L(E)=I\left(\overline{P_{l}(E)}\right)$ if and only if $\overline{P_{l}(E)}=E^{0}$.

We now prove a general result about semiprime rings, a result which will play a key role in the proof of Theorem 2.6.

Theorem 2.5. For a semiprime ring $R$ the following conditions are equivalent:

(i) $R$ is locally left artinian.

(i) $R$ is locally right artinian.

(ii) $R$ is semisimple.

(iii) For every element $a \in R$, the local ring $R_{a}$ of $R$ at $a$ is left artinian.

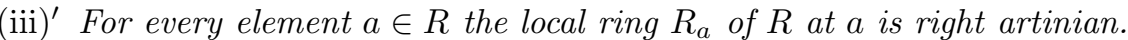

(iv) $R \cong \bigoplus_{i \in \Upsilon} \mathbb{M}_{n_{i}}\left(e_{i} R e_{i}\right)$, where $\Upsilon$ is an arbitrary set, $n_{i} \in \mathbb{N} \cup\{\infty\}$ and the $e_{i}$ 's are minimal idempotents. 
In particular, if $R$ is a ring with local units, then the conditions above are equivalent to the following ones.

(v) Every corner of $R$ is left artinian.

$(\mathrm{v})^{\prime}$ Every corner of $R$ is right artinian.

If moreover $R$ has a countable set of local units, then all conditions above are equivalent to:

(vi) $R \cong \bigoplus_{i \in \Upsilon} \mathbb{M}_{n_{i}}\left(e_{i} R e_{i}\right)$, where $\Upsilon$ is a countable set, $n_{i} \in \mathbb{N} \cup\{\infty\}$ and the $e_{i}$ 's are minimal idempotents.

Proof. (i) $\Leftrightarrow(\mathrm{i})^{\prime}$ and $(\mathrm{v}) \Leftrightarrow(\mathrm{v})^{\prime}$ follow because for every idempotent $e, e R e$ is a semiprime ring, hence it is left artinian if and only if it is right artinian.

The equivalence (iii) $\Leftrightarrow$ (iii) $^{\prime}$ can be proved similarly, utilizing the fact that every local ring at an element of a semiprime ring is also semiprime ([16, Proposition $2.1(\mathrm{i})])$.

(i) $\Rightarrow$ (ii). Let $a$ be in $R$. By the hypothesis, there exists $e^{2}=e \in R$ such that $a \in e R e$ and $e R e$ is an artinian (and semiprime) ring, hence it coincides with its socle. By [16, Proposition $2.1(\mathrm{v})], R_{a}=(e R e)_{a}$ is artinian, and applying the same result we have that $a \in \operatorname{Soc}(R)$.

(ii) $\Rightarrow$ (iv). Since the socle is a direct sum of simple rings, we may restrict our attention to the simple case, and therefore we have a primitive ring which coincides with its socle. By [12, Theorems 4.3.8 and 4.3.9] there exists a dual pair of vector spaces $\left(\Delta V, W_{\Delta}\right)$ over a division ring $\Delta$ such that $R=\mathcal{F}_{W}(V)$. By Littof's Theorem ([12, Theorem 4.3.11]), for every element $a \in \mathcal{F}_{W}(V)$ there exists an idempotent $e \in \mathcal{F}_{W}(V)$ such that $a \in e R e$ and the ring $e$ Re is isomorphic to $\mathbb{M}_{n}(\Delta)$, where $\Delta$ is a division ring isomorphic to $u R u$, for $u$ a minimal idempotent of $R$.

(iv) $\Rightarrow$ (iii). For every element $a \in R$, since the number of entries in $a$ is finite, $R_{a}$ is a direct sum of matrix rings over division rings, that is, it is left artinian.

(ii) $\Leftrightarrow$ (iii) $\Leftrightarrow$ (iii) $^{\prime}$ is $[16$, Proposition 2.1 (v)] taking into account that a semiprime ring is left artinian if and only if it is right artinian.

(ii) $\Rightarrow$ (i). Since (ii) and (iii) are equivalent, every corner is a left artinian ring. Now, if $x_{1}, \ldots, x_{n}$ are elements in $R$, use Littof's Theorem to find an idempotent $e$ in $R$ such that $x_{i} \in e R e$ for every $i=1, \ldots, n$.

(i) $\Leftrightarrow(v)$. Suppose that $R$ is locally left artinian. Take an idempotent $e \in R$ and let $f=f^{2}$ be in $R$ such that $e \in f R f$ with $f R f$ left artinian. Since $f R f$ is a unital ring, we can apply [18, Corollary (21.13)] to say that efRfe is a left artinian ring. But since $e \in f R f$ we get that $e f=f e=e$, that is, efRfe $=e R e$ is indeed left artinian. The converse is a tautology. 
(ii) $\Rightarrow$ (vi) follows the same reasoning as (ii) $\Rightarrow$ (iv) above except that we make sure that the set of homogeneous components is at most countable: If this is not the case we may then find an uncountable set $\left\{e_{\alpha}\right\}$ of pairwise orthogonal minimal idempotents. Since $R$ has a countable set of local units $\left\{f_{i}\right\}_{i=1}^{\infty}$, there is $n$ such that $f_{n} R f_{n}$ contains infinitely many pairwise orthogonal idempotents, which is impossible as $f_{n} R f_{n}$ is unital and semisimple.

(vi) $\Rightarrow(\mathrm{iv})$ is a tautology.

Recall that an abelian monoid $(M,+)$ is cancellative if $x+z=y+z$ with $x, y, z \in M$ implies $x=y$. For a $\operatorname{ring} R$, we denote by $V(R)$ the monoid of finitely generated projective left $R$-modules.

We now have all the necessary ingredients in hand to prove the main result of the section, in which we characterize the semisimple Leavitt path algebras by describing them in categorical, ring-theoretic, graph-theoretic, and explicit terms. In addition, we give a characterization of these algebras in terms of their finitely generated projective modules.

Theorem 2.6. Let $E$ be a row-finite graph. The following conditions are equivalent.

(i) $L(E)$ is semisimple.

(ii) $L(E)$ is categorically left artinian.

(ii) $^{\prime} L(E)$ is categorically right artinian.

(iii) $L(E)$ is locally left artinian.

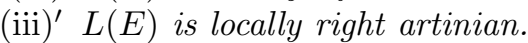

(iv) $E$ is acyclic and every infinite path ends in a sink.

(v) $L(E) \cong \bigoplus_{i \in \Upsilon} \mathbb{M}_{n_{i}}(K)$, where $\Upsilon$ is a countable set and $n_{i} \in \mathbb{N} \cup\{\infty\}$.

(vi) $L(E)$ is von Neumann regular and $V(L(E))$ is cancellative.

(vii) $L(E)$ is von Neumann regular and $V(L(E)) \cong\left(\mathbb{Z}^{+}\right) \leq \Upsilon$, that is, $V(L(E))$ is either a finite or a countable number of copies of $\mathbb{Z}^{+}$.

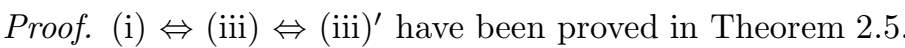

(i) $\Leftrightarrow$ (v) follows by Theorem 2.5 together with Lemma 2.3.

(ii) $\Rightarrow$ (iii) and (ii) ${ }^{\prime} \Rightarrow(\text { iii })^{\prime}$ are established in Lemma 1.6.

(v) $\Rightarrow$ (ii) and (v) $\Rightarrow(\text { ii) })^{\prime}$ are consequences of Corollary 1.3.

(iv) $\Rightarrow$ (i). By Lemma 2.4 it is enough to see that $\overline{P_{l}(E)}=E^{0}$. Suppose on the contrary that there exists $v \in E^{0}$ with $v \notin \overline{P_{l}(E)}$. Then $v$ is not a line point and clearly it cannot be a sink, so that $s^{-1}(v) \neq \emptyset$. Now, by the saturated condition, from $v \notin \overline{P_{l}(E)}$ we get that $r\left(s^{-1}(v)\right) \nsubseteq \overline{P_{l}(E)}$ so that there exists $e_{1} \in E^{1}$ with $s\left(e_{1}\right)=v$ and $r\left(e_{1}\right)=w \notin \overline{P_{l}(E)}$. We repeat this process with $w$ and we obtain some $e_{2} \in E^{1}$ with $s\left(e_{2}\right)=w$, $r\left(e_{2}\right)=x \notin \overline{P_{l}(E)}$. 
Moreover, since $E$ is acyclic by hypotheses, the vertices $\{v, w, x\}$ are indeed different. In other words, with this process we can build an infinite path $\gamma=e_{1} e_{2} e_{3} \ldots$ such that all their vertices are different and neither of them is a line point. But by hypotheses, $\gamma$ ends in sink, so that from a certain point forward, $\gamma$ does not have bifurcations (nor cycles, because $E$ is acyclic). Equivalently, past a certain point, all vertices of $\gamma$ live in $P_{l}(E)$, a contradiction.

(vi) $\Rightarrow$ (iv). Let $c$ be a cycle. We distinguish two cases.

First, suppose that $c$ has an exit $e$. We can assume that $s(e)=v$ (otherwise we would rearrange the edges of $c$ and consider the cycle $c^{\prime}$ with same edges as $c$ but based at $s(e))$. Given any positive integer $n \in \mathbb{Z}$ we claim that $L(E) c^{n}\left(c^{*}\right)^{n} \supsetneq L(E) c^{n+1}\left(c^{*}\right)^{n+1}$. The containment is clear because $c^{n+1}\left(c^{*}\right)^{n+1}=\left(c^{n+1}\left(c^{*}\right)^{n+1}\right)\left(c^{n}\left(c^{*}\right)^{n}\right)$. The sets are not equal because $c^{n}\left(c^{*}\right)^{n} \notin L(E) c^{n+1}\left(c^{*}\right)^{n+1}$ : If so, there would exist $\alpha \in L(E)$ with $c^{n}\left(c^{*}\right)^{n}=\alpha c^{n+1}\left(c^{*}\right)^{n+1}$ and then, by multiplying by $c^{n} e$ on the right hand side we would get $c^{n} e=\alpha c^{n+1} c^{*} e=0$, a contradiction since the element $c^{n} e$ is an element of the path algebra and such is always nonzero.

Now, if we consider the idempotents $e_{n}=c^{n}\left(c^{*}\right)^{n}$, then we have that in the usual order or idempotents, $e_{n} \geq e_{n+1}$ for all $n$ (meaning that $e_{n+1}=$ $\left.e_{n} e_{n+1}=e_{n+1} e_{n}\right)$. This implies that $e_{n+1}$ and $e_{n}-e_{n+1}$ are orthogonal idempotents, so that we can write $e_{n}=e_{n+1} \oplus\left(e_{n}-e_{n+1}\right)$, which, by taking classes in the monoid $V(L(E))$, gives $\left[e_{n}\right]=\left[e_{n+1}\right]+\left[e_{n}-e_{n+1}\right]$.

Moreover all idempotents are equivalent to $v$ because $v=\left(c^{*}\right)^{n} c^{n} \sim$ $c^{n}\left(c^{*}\right)^{n}=e_{n}$. So that we have $[v]=[v]+\left[e_{n}-e_{n+1}\right]$. Since by hypothesis the monoid is cancellative, we get $[0]=\left[e_{n}-e_{n+1}\right]$, or equivalently $0 \sim$ $e_{n}-e_{n+1}$, implying that $0=L(E) 0 \cong L(E)\left(e_{n}-e_{n+1}\right)$, that is, $e_{n}=e_{n+1}$, a contradiction.

Suppose now that $c$ does not have an exit. In this situation, and with a similar reasoning to that of [1, Proof of Theorem 3.11], it is not difficult to show that $v L(E) v=\left\{\sum_{\substack{i=-\infty \\ \text { finite }}}^{\infty} k_{i} c^{i}\right.$, for $\left.k_{i} \in K\right\} \cong K\left[x, x^{-1}\right]$, where we understand $c^{i}=\left(c^{*}\right)^{-i}$ for negative $i$. Now we use the hypothesis that $L(E)$ is regular von Neumann to conclude that the corner $v L(E) v \cong K\left[x, x^{-1}\right]$ is also regular, which is absurd. This means that this case cannot happen so that $E$ must be acyclic.

Suppose now that there exists an infinite path $\gamma$ which does not end in a sink, and write $v=s(\gamma)$. Since $E$ is acyclic, $\gamma$ has infinitely many bifurcations so that we can decompose it in an infinite product of paths $\gamma=\gamma_{1} \gamma_{2} \gamma_{3} \ldots$ in such a way that we have a bifurcation at $r\left(\gamma_{i}\right)$ for every $i$. We claim that the following is an infinite decreasing chain of left ideals 
of $v L(E) v$ :

$$
v L(E) v \gamma_{1} \gamma_{1}^{*} v \supsetneq v L(E) v \gamma_{1} \gamma_{2} \gamma_{2}^{*} \gamma_{1}^{*} v \supsetneq v L(E) v \gamma_{1} \gamma_{2} \gamma_{3} \gamma_{3}^{*} \gamma_{2}^{*} \gamma_{1}^{*} v \supsetneq \ldots
$$

The containments are clear as for instance $v \gamma_{1} \gamma_{2} \gamma_{2}^{*} \gamma_{1}^{*} v=$ $\left(v \gamma_{1} \gamma_{2} \gamma_{2}^{*} \gamma_{1}^{*} v\right)\left(v \gamma_{1} \gamma_{1}^{*} v\right)$. To see that they do not coincide, suppose we can write $v \gamma_{1} \gamma_{1}^{*} v=v \alpha v \gamma_{1} \gamma_{2} \gamma_{2}^{*} \gamma_{1}^{*} v$, for some $\alpha \in L(E)$. Then, as $r\left(\gamma_{1}\right)=s\left(\gamma_{2}\right)$ has a bifurcation, there exists an edge $e \in E^{1}$ with $s(e)=s\left(\gamma_{2}\right)$ but different to the first edge of $\gamma_{2}$, or equivalently, with $\gamma_{2}^{*} e=0$. Now, by multiplying on the right hand side by $\gamma_{1} e$ on the previous equation we get the following contradiction:

$$
0 \neq \gamma_{1} e=\left(v \gamma_{1} \gamma_{1}^{*} v\right) \gamma_{1} e=\left(v \alpha v \gamma_{1} \gamma_{2} \gamma_{2}^{*} \gamma_{1}^{*} v\right) \gamma_{1} e=v \alpha v \gamma_{1} \gamma_{2} \gamma_{2}^{*} e=0,
$$

Now, if we label $e_{n}=\gamma_{1} \gamma_{2} \ldots \gamma_{n} \gamma_{n}^{*} \ldots \gamma_{2}^{*} \gamma_{1}^{*} v$, the infinite chain above implies that $\left\{e_{n}\right\}_{n=1}^{\infty}$ is an infinite decreasing family of idempotents verifying the same properties to that of the previous case of a cycle with an exit, and therefore leading to a contradiction.

(v) $\Rightarrow$ (vii) and (vii) $\Rightarrow$ (vi) are well-known.

We conclude this section by giving a structure theorem for Leavitt path algebras with essential socle. The key point is the fact that the Leavitt path algebra $L_{K}(E)$ is an algebra of right quotients of the path algebra $K E$ [20]. That this class of Leavitt path algebras does not coincide with the class of semisimple Leavitt path algebras is seen by analyzing the Toeplitz algebra, i.e., the Leavitt path algebra whose graph is the following (see [20]):

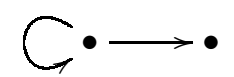

By $\operatorname{RCFM}(K)$ we understand the algebra of infinite matrices with finite row and columns.

Theorem 2.7. For a graph $E$ the following conditions are equivalent.

(i) The Leavitt path algebra $L(E)$ has essential socle.

(ii) Every vertex connects to a line point.

(iii) $L(E)$ satisfies:

$$
\begin{aligned}
\left(\bigoplus_{i \in \Upsilon_{1}}\right. & \left.\mathbb{M}_{n_{i}}(K)\right) \oplus\left(\bigoplus_{j \in \Upsilon_{2}} \mathbb{M}_{m_{j}}(K)\right) \subseteq L(E) \\
& \subseteq\left(\prod_{i \in \Upsilon_{1}} \mathbb{M}_{n_{i}}(K)\right) \oplus\left(\prod_{j \in \Upsilon_{2}} \operatorname{RCFM}(K)\right),
\end{aligned}
$$

where $n_{i} \in \mathbb{N}$ and $m_{j}=\infty$.

Proof. (i) $\Leftrightarrow$ (ii) is [20, Theorem 4.3].

(i) $\Rightarrow$ (iii). By $\left[8\right.$, Theorem 4.2], $S o c(L(E))=I(H)$, for $H=\overline{P_{l}(E)}$, and by [6, Lemma 1.2], $I(H) \cong L\left({ }_{H} E\right)$. Now apply Theorem 2.6 and [20, Corollary 4.6] to obtain the result. 
(iii) $\Rightarrow$ (i). Denote by $R$ and $Q$ the algebras in the statement such that $R \subseteq L(E) \subseteq Q$, and observe that $Q=Q_{\max }^{r}(R)$. Take a nonzero ideal $I$ of $L(E)$. Since $L(E)$ is an algebra of right quotients of $R, I \cap R \neq 0$ ([16, Proposition 3.1 (i)]). For a nonzero $u \in I \cap R$, by [16, Proposition $3.2(\mathrm{v})]$ we have that $L(E)_{u}$ is an algebra of right quotients of $R_{u}$, which is a semisimple and artinian algebra, hence $L(E)_{u}=R_{u}$ and therefore $u \in \operatorname{Soc}(L(E))$, by [16, Proposition $2.1(\mathrm{v})]$.

\section{Noetherian Leavitt PATH Algebras}

Our investigation of the unital artinian Leavitt path algebras (Theorem 2.2) led us naturally to the study of the not-necessarily-unital categorically artinian Leavitt path algebras, an effort which was completed in Theorem 2.6. With this in mind, a natural next step in our study is to investigate the structure of the categorically noetherian Leavitt path algebras. This is the main goal of the current section. In symmetry with the artinian case, these Leavitt path algebras turn out to be the locally noetherian Leavitt path algebras.

The noetherian counterpart to Theorem 2.2 was provided in [5]; we present it here for completeness. We recall that there is a natural $\mathbb{Z}$-grading on $L(E)$, see e.g. [5, Section 1] or [21, Section 3.3]. Additionally, a graph $E$ is said to satisfy Condition (NE) if no cycle in $E$ has an exit.

Theorem 3.1. ([5, Theorem 3.8 and Theorem 3.10]) Let E be a finite graph (in other words, let $L(E)$ be unital). The following conditions are equivalent.

(i) $L(E)$ is left noetherian.

(i) $)^{\prime} L(E)$ is right noetherian.

(ii) $L(E) \cong\left(\bigoplus_{i=1}^{l} \mathbb{M}_{m_{i}}\left(K\left[x, x^{-1}\right]\right)\right) \oplus\left(\bigoplus_{j=1}^{l^{\prime}} \mathbb{M}_{n_{j}}(K)\right)$ for some positive integers $m_{1}, \ldots, m_{l}, n_{1}, \ldots, n_{l^{\prime}}$.

(iii) E satisfies Condition (NE).

(iv) $L(E)$ is locally finite, that is, the graded component $(L(E))_{n}$ is finite dimensional for each $n \in \mathbb{Z}$.

With Theorem 3.1 in mind, we can cast the main goal of this section as the natural destination of a journey consisting of two different routes: extending the categorically artinian condition to categorically noetherian, and/or extending the unital noetherian condition to the appropriate analog of the noetherian condition for non-unital rings.

The following result is a generalization of [5, Theorem 3.8]. The proof consists of splitting the problem into several pieces, some of which rely on previously studied situations (as the semisimple case of Theorem 2.6), or on careful generalizations to the non unital setting of known results (as the locally finite just infinite case stated in [5, Theorem 3.3]). This approach 
has been chosen to avoid as much as possible the multiple technicalities of dealing with bases.

Lemma 3.2. Let $H$ be a hereditary subset of $E^{0}$, for a graph $E$. Then

$$
I(H)=\left\{\sum k \alpha \beta^{*} \text {, with } k \in K, \alpha, \beta \text { paths such that } r(\alpha)=r(\beta) \in H\right\} .
$$

Proof. Denote by $J$ the set $\left\{\sum k \alpha \beta^{*} \mid k \in K, \alpha, \beta\right.$ are paths and $r(\alpha)=r(\beta) \in H\}$. The containment $J \subseteq I(H)$ is clear. For the converse, consider $\mu, \nu, \alpha, \beta$ paths in $L(E)$, and $u \in H$ such that $\mu \nu^{*} u \alpha \beta^{*} \neq 0$. By [21, Lemma 3.1], $\mu \nu^{*} u \alpha \beta^{*}$ is $\mu \alpha^{\prime} \beta^{*}$ if $\alpha=\nu \alpha^{\prime}$ or $\mu \nu^{\prime *} \beta^{*}$ if $\nu=\alpha \nu^{\prime}$. Note that $\alpha=\nu \alpha^{\prime}, u=s(\alpha)$ and $H$ hereditary imply $r\left(\alpha^{\prime}\right) \in H$, hence $\mu \alpha^{\prime} \beta^{*} \in J$. In the second case, $\nu=\alpha \nu^{\prime}, u=s(\alpha)$ and $H$ hereditary imply $s\left(\nu^{\prime *}\right)=r\left(\nu^{\prime}\right) \in H$, hence $\mu r\left(\nu^{\prime}\right) \nu^{\prime *} \beta^{*} \in J$, therefore $I(H) \subseteq J$.

The notion of $C_{n}$-comet was introduced in [5] to describe the locally finite Leavitt path algebras. The role of the cycle $C_{n}$ within a $C_{n}$-comet is similar to that played by sinks in more general graphs. If a graph $E$ is a $C_{n}$-comet, then its associated Leavitt path algebra is isomorphic to $\mathbb{M}_{n}\left(K\left[x, x^{-1}\right]\right)$. Since $C_{n}$-comets have a finite number of vertices, it is natural to generalize this concept to the case of an infinite (numerable) set of vertices.

Definition 3.3. We say that a graph $E$ is a comet if it has exactly one cycle $c, T(v) \cap c^{0} \neq \emptyset$ for every vertex $v \in E^{0}$, and every infinite path ends in the cycle $c$.

Remark 3.4. As stated before, when we had a finite $C_{n}$-comet graph $E$, [5, Theorem 3.3] gave that $L(E) \cong \mathbb{M}_{n}\left(K\left[x, x^{-1}\right]\right)$, where $n$ was the number of paths in $E$ which ended in the cycle $c$ but did not contain it completely. Our aim is to generalize this result to infinite (comet) graphs $F$ so that we can obtain the isomorphism $L(F) \cong \mathbb{M}_{\infty}\left(K\left[x, x^{-1}\right]\right)$. In order to achieve this we have to add the extra condition "every infinite path ends in the cycle $c "$, as the following example shows

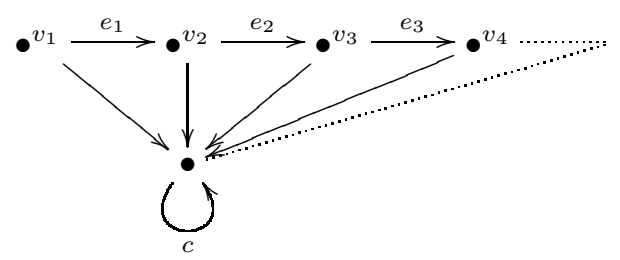

This graph $F$ verifies that $c$ is its only cycle and $T(v) \cap c^{0} \neq \emptyset$ for every vertex $v \in F^{0}$. However, as it will be proved later, if we had $L(F) \cong$ $\mathbb{M}_{\infty}\left(K\left[x, x^{-1}\right]\right)$, then Theorem 3.8 would yield that every infinite path in $F$ ends either in a sink or in a cycle. But the infinite path $\gamma=e_{1} e_{2} e_{3} \ldots$ does not end neither in a sink nor in a cycle. 
In any case, when $E$ is finite, Definition 3.3 agrees with that of $C_{n}$-comet given in [5].

Proposition 3.5. Let $E$ be a graph which is a comet. Then the Leavitt path algebra $L(E)$ is isomorphic to $\mathbb{M}_{n}\left(K\left[x, x^{-1}\right]\right)$, where $n \in \mathbb{N}$ if $E$ is finite, or $n=\infty$ otherwise.

Proof. We can adapt [5, Theorem 3.3] to our situation. Concretely, let $c$ be the cycle in $E, v$ a vertex at which the cycle $c$ is based and consider $\left\{p_{i}\right\}$ the (perhaps infinite) family of all paths in $E$ which end in $v$ but do not contain the cycle $c$. Let $n \in \mathbb{N} \cup\{\infty\}$ be the number of all such paths. Denote by $N$ the set $\{1, \ldots, n\}$ when $n$ is finite and $N=\mathbb{N}$ when $n=\infty$. Consider the family $\mathcal{B}:=\left\{p_{i} c^{k} p_{j}^{*}\right\}_{i, j \in N, k \in \mathbb{N}}$ of monomials in $L(E)$ where we understand $c^{0}=v$ and $c^{n}=\left(c^{*}\right)^{-n}$ for negative $n$.

As in [5, Theorem 3.3], we can show that $\mathcal{B}$ is a linearly independent set. We will prove that $\mathcal{B}$ generates $L(E)$ as a $K$-vector space. First, note that since $E$ is a comet, then $T(v)$ is a finite set for every $v \in c^{0}$. Not only is this true for any vertex on the cycle $c$ but for any vertex in $E$ as follows: Suppose on the contrary that there exists $w \in E$ with $|T(w)|=\infty$. In particular, $w$ does not lie on the cycle. As $E$ is row-finite, we are able to find and edge $e_{1}$ in $E$ with $s\left(e_{1}\right)=w$ and $v_{1}:=r\left(e_{1}\right)$ such that $\left|T\left(v_{1}\right)\right|=\infty$. Again $v_{1}$ does not lie on the cycle. Repeating this process, we find an infinite path such that neither of its vertices lie on $c$, which contradicts the fact that every infinite path in $E$ ends in the cycle $c$.

Take an arbitrary element $\sum_{i} k_{i} \alpha_{i} \beta_{i}^{*}$ of $L(E)$, where $\alpha_{i}, \beta_{i}$ are paths in $E$ and $k_{i} \in K$. Consider the set $\left\{r\left(\alpha_{i}\right)\right\}$. Some of these vertices could lie on the cycle $c$, in which case we leave the corresponding monomial as is. For those monomials $\alpha_{k} \beta_{k}^{*}$ whose $\left\{r\left(\alpha_{k}\right)\right\}$ is not on $c$, we proceed as in [4, Proof of Proposition 3.5] by using relation (4) to expand it as

$$
\alpha_{k} \beta_{k}^{*}=\sum_{\left\{e \in E^{1}: s(e)=r\left(\alpha_{k}\right)\right\}} \alpha_{k} e e^{*} \beta_{k}^{*}=\sum_{\left\{e \in E^{1}: s(e)=r\left(\alpha_{k}\right)\right\}}\left(\alpha_{k} e\right)\left(\beta_{k} e\right)^{*} .
$$

As we have just proved that the tree of any vertex is finite, so will be this process of expanding these monomials until reaching vertices of $c$.

Consider now a monomial $\alpha_{k} \beta_{k}^{*}$ with $r\left(\alpha_{k}\right) \in c^{0}$. Let $t$ be the subpath of $c$ with $s(t)=r\left(\alpha_{k}\right)$ and $r(t)=v$. Since $c$ does not have exits then $\alpha_{k} \beta_{k}^{*}=\alpha_{k} t t^{*} \beta_{k}^{*}=\left(\alpha_{k} t\right)\left(\beta_{k} t\right)^{*}=\alpha \beta^{*}$, where $\alpha$ and $\beta$ are paths in $E$ that end in $v$. Finally, since $E$ is a comet, we can always factor some powers of $c$ out of $\alpha$ and $\beta$ so that there exist integers $m, n$ such that $\alpha=p_{i} c^{m}$ and $\beta=p_{j} c^{n}$ for some paths $p_{i}, p_{j}$ which do not contain the path $c$. Hence, we obtain that $\alpha_{k} \beta_{k}^{*}=p_{i} c^{m-n} p_{j}^{*} \in \mathcal{B}$. This proves that $\mathcal{B}$ is a $K$-generator of $L(E)$. 
Now, by defining $\phi: L(E) \rightarrow \mathbb{M}_{n}\left(K\left[x, x^{-1}\right]\right)$ on the basis by setting $\phi\left(p_{i} c^{k} p_{j}^{*}\right)=x^{k} e_{i j}$ for $e_{i j}$ the $(i, j)$-matrix unit, then again one easily checks that $\phi$ is a $K$-algebra isomorphism.

For a graph $E$, denote by $P_{c}(E)$ the set of vertices in the cycles without exits of $E$.

Proposition 3.6. Let $E$ be a graph. Then:

(i) $I\left(P_{l}(E)\right) I\left(P_{c}(E)\right)=0$.

(ii) $I\left(P_{c}(E)\right)=\bigoplus_{j \in \Upsilon} I\left(P_{c_{j}}(E)\right)$, where $\Upsilon$ is a countable set and $\left\{c_{j}\right\}_{j \in \Upsilon}$ is the set of all different cycles without exits of $E$ (and by abuse of notation we identify two cycles that have the same vertices).

(iii) $P_{c}(E)$ is hereditary and if $H$ denotes the saturated closure of $P_{c}(E)$, we have that

$I\left(P_{c}(E)\right)=I(H) \cong L\left({ }_{H} E\right) \cong \bigoplus_{i \in \Upsilon_{1}} \mathbb{M}_{n_{i}}\left(K\left[x, x^{-1}\right]\right) \oplus \bigoplus_{j \in \Upsilon_{2}} \mathbb{M}_{m_{j}}\left(K\left[x, x^{-1}\right]\right)$, where $\Upsilon_{1}$ and $\Upsilon_{2}$ are countable sets, $n_{i} \in \mathbb{N}$ and $m_{j}=\infty$.

Proof. We will use Lemma 3.2 implicitly. This can be done because $P_{c}(E)$ is, clearly, a hereditary set.

(i). Suppose that we have nonzero monomials $\alpha \beta^{*}$ and $\gamma \delta^{*}$ with $r(\alpha) \in$ $P_{l}(E)$ and such that there exits a cycle without exits based at $r(\gamma)$. If $\alpha \beta^{*} \gamma \delta^{*} \neq 0$, two possibilities can occur: If $\gamma=\beta p$ for some path $p$, then this implies that there is a cycle based at $r(p)=r(\gamma) \in T(r(\beta))$, that is: $r(\beta)=r(\alpha) \notin P_{l}(E)$. So this possibility cannot happen. Thus, $\beta=\gamma q$ for some path $q$. Now, since $r(\gamma)$ lies on a cycle which has no exists, $r(q)=$ $r(\beta)=r(\alpha)$ lies on this same cycle, contradicting again the hypothesis.

(ii). To shorten the notation, write: $\mathcal{J}=I\left(P_{c}(E)\right)$ and $\mathcal{J}_{j}=I\left(P_{c_{j}}(E)\right)$.

Consider monomials $\gamma \delta^{*}$ with $r(\delta) \in\left(c_{j}\right)^{0}$ and $\sigma \tau^{*} \in \mathcal{J}$. Since the cycles $c_{j}$ have no exits, they are disjoint and then, similar arguments to that of the previous paragraph show that $\gamma \delta^{*} \sigma \tau^{*}, \sigma \tau^{*} \gamma \delta^{*} \in \mathcal{J}_{c_{j}}$. Moreover, these arguments also yield that if $\sigma \tau^{*} \in \mathcal{J}_{c_{k}}$ with $j \neq k$, then $\gamma \delta^{*} \sigma \tau^{*}=\sigma \tau^{*} \gamma \delta^{*}=$ 0 . Thus, $\left\{\mathcal{J}_{c_{j}}\right\}$ is indeed a family of orthogonal ideals of $\mathcal{J}$.

To show that $\mathcal{J}=\sum_{j} \mathcal{J}_{j}$ apply Lemma 3.2 to $H=\cup_{j} c_{j}^{0}$, which is a hereditary set since the considered cycles have no exits.

(iii). $I\left(P_{c}(E)\right)=I(H)$ follows by $\left[9\right.$, Lemma 2.1] and $I(H) \cong L\left({ }_{H} E\right)$ by [6, Lemma 1.2]. The same results applied to $c_{j}$ instead of $c$ imply $I\left(P_{c_{j}}(E)\right)=I\left(H_{j}\right) \cong L\left(H_{j} E\right)$, for $H_{j}$ the saturated closure of $P_{c_{j}}$. By the definition of $H_{j}$, and since $c_{j}$ has no exits, every vertex in $H_{j}$ connects to $c_{j}$. The same can be said about $H_{j} E$, where $c_{j}$ can be seen as its only cycle. Now suppose that $\gamma$ is an infinite path in $H_{j} E$. Again, by the way this graph is constructed, there must exist a finite path $p$ and an 
infinite path $\beta$ such that $\gamma=p \beta$, with $\beta$ being completely contained in $E_{H_{j}}$. Suppose that $\beta$ does not end in the cycle $c_{j}$. This, together with the fact that $c_{j}$ does not have exits, yield that $\beta^{0} \cap c_{j}^{0}=\emptyset$. On the other hand, because $\beta^{0} \subseteq H_{j}$ we can consider $m$ to be the minimum $n$ such that $\Lambda_{n}\left(c_{j}^{0}\right) \cap \beta^{0} \neq \emptyset$. Now, $\beta^{0} \cap c_{j}^{0}=\emptyset$ implies that $m>0$ so that there exists $w \in\left\{v \in\left(E_{H_{j}}\right)^{0} \mid \emptyset \neq r\left(s^{-1}(v)\right) \subseteq \Lambda_{m-1}\left(c_{j}^{0}\right)\right\} \cap \beta^{0}$. As $\beta$ is infinite, there is an edge $e$ in $\beta$ such that $s(e)=w$ and $r(e) \in \beta^{0}$. This contradicts the minimality of $m$. Therefore $\beta$ ends in the cycle $c$, and consequently $\gamma$. Hence, ${ }_{H_{j}} E$ is a comet. Apply Proposition 3.5 and (ii) to obtain the result.

Proposition 3.7. Let $E$ be a graph satisfying Condition (NE) and such that every infinite path ends either in a sink or in a cycle. Then $L(E) \cong$ $\bigoplus_{i \in \Upsilon_{1}} \mathbb{M}_{n_{i}}(K) \oplus \bigoplus_{j \in \Upsilon_{2}} \mathbb{M}_{m_{j}}\left(K\left[x, x^{-1}\right]\right)$, where $\Upsilon_{1}$ and $\Upsilon_{2}$ are countable sets and $n_{i}, m_{j} \in \mathbb{N} \cup\{\infty\}$.

Proof. Denote by $\mathcal{I}$ and $\mathcal{J}$ the ideals generated by $P_{l}(E)$ and $P_{c}(E)$, respectively. We will apply in what follows Lemma 3.2 whenever we need to consider an element in $\mathcal{I}$ or in $\mathcal{J}$.

We show first that $L(E)=\mathcal{I}+\mathcal{J}$. Suppose that there exists $x \in L(E) \backslash$ $(\mathcal{I}+\mathcal{J})$. If we write $x=\sum_{i} k_{i} p_{i} q_{i}^{*}$, then we can find $j$ such that $p_{j} q_{j}^{*} \in$ $L(E) \backslash(\mathcal{I}+\mathcal{J})$. In particular $v_{1}:=r\left(p_{j}\right) \notin P_{l}(E)$ (and therefore it is not a sink) as $p_{j} v_{1} q_{j}^{*} \notin \mathcal{I}$. Furthermore, $v_{1}$ cannot lie on a cycle as $p_{j} v_{1} q_{j}^{*} \notin \mathcal{J}$.

Now, if $r\left(s^{-1}\left(v_{1}\right)\right) \subseteq \mathcal{I}+\mathcal{J}$, then by relation (4) we would have that

$$
p_{j} q_{j}^{*}=\sum_{\{e: s(e)=v\}} p_{j} e e^{*} q_{j}^{*}=\sum_{\{e: s(e)=v\}}\left(p_{j} e\right) r(e)\left(q_{j} e\right)^{*} \in \mathcal{I}+\mathcal{J},
$$

which is impossible. This shows that there exists $e_{1} \in E^{1}$ with $s\left(e_{1}\right)=v_{1}$ and $v_{2}:=r\left(e_{1}\right) \notin \mathcal{I}+\mathcal{J}$. From $v_{2} \notin \mathcal{I}$ we deduce that $v_{2} \notin P_{l}(E)$ and from $v_{2} \notin \mathcal{J}$ we get that $v_{2}$ does not lie on a cycle.

We repeat this process and then we are able to find an infinite path $e_{1} e_{2} \ldots$ such that neither of their vertices are line points nor lie on a cycle, a contradiction to our hypothesis. Now, since $L(E)$ has local units, the sum is direct by Proposition 3.6 (i). Hence, we have $L(E)=\mathcal{I} \oplus \mathcal{J}$.

Note that $\mathcal{I}=I\left(P_{l}(E)\right) \cong L(E) / I\left(P_{c}(E)\right) \cong L(E / H)$, for $H$ the saturated closure of $P_{c}(E)$, and that this last isomorphism is given by [9, Lemma 2.3 (i)]. This means that $\mathcal{I}$, which is the ideal generated by the line points in $E^{0}$, that is, the socle of $L(E)$, is a Leavitt path algebra which coincides with its socle. By Theorem 2.6 we have

$$
\mathcal{I} \cong \bigoplus_{i \in \Upsilon_{1}} \mathbb{M}_{n_{i}}(K)
$$

where $\Upsilon_{1}$ is a countable set and $n_{i} \in \mathbb{N} \cup\{\infty\}$. 
On the other hand, by Proposition 3.6 (iii) we have that

$$
\mathcal{J} \cong \bigoplus_{j \in \Upsilon_{2}} \mathbb{M}_{m_{j}}\left(K\left[x, x^{-1}\right]\right)
$$

where $\Upsilon_{2}$ is a countable set and $m_{j} \in \mathbb{N} \cup\{\infty\}$. We now put together these two pieces to get the result.

Finally we are in position to prove our main result of this section, in which we present the categorically noetherian Leavitt path algebras by describing them in categorical, ring-theoretic, graph-theoretic, and explicit terms.

Theorem 3.8. Let $E$ be a graph. The following conditions are equivalent.

(i) $L(E)$ is categorically left noetherian.

$(\mathrm{i})^{\prime} L(E)$ is categorically right noetherian.

(ii) $L(E)$ is locally left noetherian.

(ii) $L(E)$ is locally right noetherian.

(iii) E satisfies Condition (NE) and every infinite path ends either in a sink or in a cycle.

(iv) $L(E) \cong \bigoplus_{i \in \Upsilon_{1}} \mathbb{M}_{n_{i}}(K) \oplus \bigoplus_{j \in \Upsilon_{2}} \mathbb{M}_{m_{j}}\left(K\left[x, x^{-1}\right]\right)$, where $\Upsilon_{1}$ and $\Upsilon_{2}$ are countable sets and $n_{i}, m_{j} \in \mathbb{N} \cup\{\infty\}$.

Proof. (i) $\Rightarrow$ (ii) and (i) $\Rightarrow(\text { ii) })^{\prime}$ were established in Lemma 1.5.

(iv) $\Rightarrow$ (i) and (iv) $\Rightarrow$ (i) follow from Corollary 1.3.

(ii) $\Rightarrow$ (iii). Suppose that $c$ is a cycle in $E$ with an exit. Let $v$ be the vertex in which the cycle is based at. With similar computations to that of $[5$, Proof of (ii) $\Rightarrow$ (iii) in Theorem 3.10] we can check that the following

$$
v L(E) v\left(v-c c^{*}\right) \subsetneq v L(E) v\left(v-c^{2}\left(c^{*}\right)^{2}\right) \subsetneq \ldots
$$

is an infinite ascending chain of left ideals of $v L(E) v$, which is a contradiction to the locally left noetherian hypothesis.

This shows that $E$ satisfies Condition (NE). Suppose now that $\gamma$ is an infinite path which does not end neither in a sink nor in a cycle. In this situation $\gamma$ cannot contain any closed path as follows: If $\gamma=\gamma_{1} p \gamma_{2}$ with $p$ being a closed path, then, as we have just shown that $E$ satisfies (NE), $p$ must be in fact a cycle and $\gamma_{2}=p p p \ldots$, so that $\gamma$ does end in a cycle, contrary to hypothesis. Now, since $\gamma$ does not end in a sink either (and does not contain cycles), $\gamma$ has infinitely many bifurcations so that we can write $\gamma=\gamma_{1} \gamma_{2} \gamma_{3} \ldots$ for $\gamma_{i}$ paths such that $r\left(\gamma_{i}\right)$ is a bifurcation for all $i$. Then we have the chain of left ideals of $v L(E) v$ given by

$$
v L(E) v\left(v-\gamma_{1} \gamma_{1}^{*}\right) \subsetneq v L(E) v\left(v-\gamma_{1} \gamma_{2} \gamma_{2}^{*} \gamma_{1}^{*}\right) \subsetneq \ldots
$$

Indeed, the containments follow from the easily checked equation

$$
v-\gamma_{1} \ldots \gamma_{n} \gamma_{n}^{*} \ldots \gamma_{1}^{*}=\left(v-\gamma_{1} \ldots \gamma_{n} \gamma_{n}^{*} \ldots \gamma_{1}^{*}\right)\left(v-\gamma_{1} \ldots \gamma_{n+1} \gamma_{n+1}^{*} \ldots \gamma_{1}^{*}\right)
$$


They are proper because if not, we would be able to write

$$
v-\gamma_{1} \ldots \gamma_{n+1} \gamma_{n+1}^{*} \ldots \gamma_{1}^{*}=(v \alpha v)\left(v-\gamma_{1} \ldots \gamma_{n} \gamma_{n}^{*} \ldots \gamma_{1}^{*}\right)
$$

for some $\alpha \in L(E)$, which after multiplying on the right by $\gamma_{1} \ldots \gamma_{n}$ gives

$$
\gamma_{1} \ldots \gamma_{n}-\gamma_{1} \ldots \gamma_{n} \gamma_{n+1} \gamma_{n+1}^{*}=(v \alpha v)\left(\gamma_{1} \ldots \gamma_{n}-\gamma_{1} \ldots \gamma_{n}\right)=0 \text {, }
$$

or equivalently, $\gamma_{1} \ldots \gamma_{n}=\gamma_{1} \ldots \gamma_{n} \gamma_{n+1} \gamma_{n+1}^{*}$. Find $e$ an exit at $r\left(\gamma_{n}\right)$ and multiply by $e$ on the right of the previous equation to reach a contradiction.

(ii) ${ }^{\prime} \Rightarrow$ (iii) is proved analogously.

(iii) $\Rightarrow$ (iv) is Proposition 3.7.

Remark 3.9. Recently, the notion of Leavitt path algebra for not necessarily row-finite graphs has been introduced in [3]. The only difference when we consider a graph with infinite emitters is that the corresponding Leavitt path algebra lacks, by definition, the relation (4) at them. In [3] the authors showed that with this broader definition, the family of Leavitt path algebras is properly enlarged.

Throughout this paper we have focused our attention on the row-finite case only because for the row-infinite case we never obtain neither a locally (left) artinian nor a locally (left) noetherian Leavitt path algebra, as is proved in what follows:

Suppose that there exists a vertex $v$ in $E$ which emits infinitely many edges $\left\{e_{i}\right\}_{i=1}^{\infty}$ (in other words, $v$ is an infinite emitter). Consider the infinite ascending chain of left ideals of $v L(E) v$ given by:

$$
v L(E) v e_{1} e_{1}^{*} v \subsetneq \bigoplus_{i=1}^{2} v L(E) v e_{i} e_{i}^{*} v \subsetneq \bigoplus_{i=1}^{3} v L(E) v e_{i} e_{i}^{*} v \subsetneq \ldots
$$

The containments are proper: suppose that $v e_{j} e_{j}^{*} v e_{j} e_{j}^{*} v=e_{j} e_{j}^{*} \in$ $\bigoplus_{i=1}^{j-1} v L(E) v e_{i} e_{i}^{*} v$. Write $e_{j} e_{j}^{*}=\sum_{i=1}^{j-1} v \alpha_{i} v e_{i} e_{i}^{*} v$, for some $\alpha_{i} \in L(E)$. Then, by multiplying on the right hand side by $e_{j} e_{j}^{*}$, we get a contradiction. Thus, $L(E)$ is not locally left noetherian.

Analogously, the following is an infinite descending chain of left ideals inside $v L(E) v$ :

$$
\bigoplus_{i=1}^{\infty} v L(E) v e_{i} e_{i}^{*} v \supsetneq \bigoplus_{i=2}^{\infty} v L(E) v e_{i} e_{i}^{*} v \supsetneq \bigoplus_{i=3}^{\infty} v L(E) v e_{i} e_{i}^{*} v \supsetneq \ldots
$$

and therefore $L(E)$ is not locally left artinian.

The following result is the analog to Theorem 2.7 by changing "connects to a line point" to "connects to a cycle without exits". Again the key tool is the use of the fact that the Leavitt path algebra $L_{K}(E)$ is an algebra of right quotients of the path algebra $K E$ (see [20]). 
Theorem 3.10. For a graph $E$ the following conditions are equivalent:

(i) Every vertex of $E^{0}$ connects to a cycle without exits.

(ii) $I\left(P_{c}(E)\right)$ is an essential (graded) ideal.

(iii) There exist countable sets $\Upsilon_{1}$ and $\Upsilon_{2}$ such that

$$
\begin{aligned}
& \bigoplus_{i \in \Upsilon_{1}} \mathbb{M}_{n_{i}}\left(K\left[x, x^{-1}\right]\right) \oplus \bigoplus_{j \in \Upsilon_{2}} \mathbb{M}_{m_{j}}\left(K\left[x, x^{-1}\right]\right) \subseteq \\
& L(E) \subseteq \\
& \prod_{i \in \Upsilon_{1}} \mathbb{M}_{n_{i}}\left(K\left[x, x^{-1}\right]\right) \oplus \prod_{j \in \Upsilon_{2}} R C F M\left(K\left[x, x^{-1}\right]\right),
\end{aligned}
$$

where $n_{i} \in \mathbb{N}$ and $m_{j}=\infty$.

Proof. (i) $\Rightarrow$ (ii). Let $y$ be a nonzero element of $L(E)$. By [8, Proposition 3.1] there exist $v \in E^{0}$ and $\gamma, \mu \in L(E)$ such that $0 \neq \gamma y \mu=k v \in K v$, or there exists a cycle $c$ without exits, and $w \in c^{0}$, such that $0 \neq \gamma y \mu \in$ $w L(E) w \subseteq I\left(P_{c}(E)\right)$. In the first case, since every vertex connects to a cycle without exits, there exist $u \in P_{c}(E)$ and a path $\alpha \in E^{*}$ satisfying $s(\alpha)=v$ and $r(\alpha)=u$. Then $u=\alpha^{*} \alpha=\alpha^{*} v \alpha=k^{-1} \alpha^{*} \gamma y \mu \alpha \in I\left(P_{c}(E)\right)$. This shows that $I\left(P_{c}(E)\right)$ is an essential ideal of $L(E)$.

(ii) $\Rightarrow$ (i). Take a vertex $v \in E^{0}$. Since $I\left(P_{c}(E)\right)$ is essential as an ideal and $L(E)$ is semiprime, $I\left(P_{c}(E)\right)$ is a semiprime essential left ideal. Use this fact and Lemma 3.2 to find $\alpha \in L(E), \alpha_{i}, \beta_{i} \in E^{*}, k_{i} \in K$ and $w_{i} \in P_{c}(E)$, for $i=1, \ldots, n$ and $n \in \mathbb{N}$, such that $0 \neq \alpha v=\sum_{i=1}^{n} k_{i} \alpha_{i} w_{i} \beta_{i}^{*}$. This means that for some $i, 0 \neq w_{i} \beta_{i}^{*} v$, that is, $\beta_{i}$ is a path that joins $v$ to $w_{i}$.

(ii) $\Rightarrow$ (iii). By [20, Proposition 4.1], $L(E)$ is nonsingular, hence $I\left(P_{c}(E)\right)$ is a graded-dense ideal of $L(E)$. Therefore, $Q_{g r-\max }^{l}\left(I\left(P_{c}(E)\right)\right)=$ $Q_{g r-\max }^{l}(L(E))$, where $Q_{g r-\max }^{l}(R)$ denotes the graded-maximal algebra of left quotients of an algebra $R$ (see [11] for the definition and the development of the notion of graded algebra of left-quotients). Apply this and Proposition 3.6 (iii) to reach (iii).

(iii) $\Rightarrow$ (ii). Denote by $R$ and $Q$ the algebras in the statement such that $R \subseteq L(E) \subseteq Q$, and observe that $Q=Q_{g r-\max }^{l}(R)$. Use Proposition 3.6 (iii) to get $I\left(P_{c}(E)\right) \cong R \subseteq L(E)$. Thus, we have that $L(E)$ is a graded algebra of left quotients of $I\left(P_{c}(E)\right)$. This, and the fact of being $L(E)$ semiprime, show that $I\left(P_{c}(E)\right)$ is an essential ideal of $L(E)$.

Finally, adapting the arguments in Theorems 2.7 and 3.10, we can prove the following.

Theorem 3.11. For a graph $E$ the following conditions are equivalent:

(i) Every vertex of $E^{0}$ connects to a line point or to a cycle without exits. 
(ii) $\operatorname{Soc}(L(E)) \oplus I\left(P_{c}(E)\right)$ is an essential (graded) ideal.

(iii) There exist countable sets $\Upsilon_{i}$ for $i=1, \ldots, 4$ such that

$$
\begin{aligned}
& \bigoplus_{i \in \Upsilon_{1}} \mathbb{M}_{n_{i}}(K) \oplus \bigoplus_{j \in \Upsilon_{2}} \mathbb{M}_{m_{j}}(K) \oplus \bigoplus_{i^{\prime} \in \Upsilon_{3}} \mathbb{M}_{n_{i^{\prime}}^{\prime}}\left(K\left[x, x^{-1}\right]\right) \oplus \bigoplus_{j^{\prime} \in \Upsilon_{4}} \mathbb{M}_{m_{j^{\prime}}^{\prime}}\left(K\left[x, x^{-1}\right]\right) \subseteq \\
& L(E) \subseteq \\
& \prod_{i \in \Upsilon_{1}} \mathbb{M}_{n_{i}}(K) \oplus \prod_{j \in \Upsilon_{2}} R C F M(K) \oplus \prod_{i^{\prime} \in \Upsilon_{3}} \mathbb{M}_{n_{i^{\prime}}}\left(K\left[x, x^{-1}\right]\right) \oplus \prod_{j^{\prime} \in \Upsilon_{4}} \operatorname{RCFM}\left(K\left[x, x^{-1}\right]\right), \\
& \quad \text { where } n_{i}, n_{i^{\prime}}^{\prime} \in \mathbb{N} \text { and } m_{j}, m_{j^{\prime}}^{\prime}=\infty .
\end{aligned}
$$

\section{ACKNOWLEDGMENTS}

The authors would like to thank P. Ara and E. Pardo for their valuable discussions during the preparation of this paper. The first author was supported by a Sabbatical Program by the MEC (SAB2005-0028) and the second author by a Centre de Recerca Matemàtica Fellowship within the Research Programme "Discrete and Continuous Methods on Ring Theory". The third author was partially supported by the DGI MEC-FEDER through Project MTM2005-00934 and by the Comissionat per Universitats i Recerca de la Generalitat de Catalunya, the second and fourth authors by the Spanish MEC and Fondos FEDER jointly through projects MTM200406580-C02-02 and by the Junta de Andalucía PAI project FQM-1215, and all four authors by the "Plan Andaluz de Investigación y Desarrollo Tecnológico" project FQM-336. Part of this work was carried out during visits of the first, second and fourth authors to the Universitat Autònoma de Barcelona and Centre de Recerca Matemàtica, and of the first, second and third authors to the Universidad de Málaga. They thank these host centers for their warm hospitality and support.

\section{REFERENCES}

1. G. Abrams, G. Aranda Pino, The Leavitt path algebra of a graph, J. Algebra 293 (2) (2005), 319-334.

2. G. Abrams, G. Aranda Pino, Purely infinite simple Leavitt path algebras, J. Pure Appl. Algebra 207 (3) (2006), 553-563.

3. G. Abrams, G. Aranda Pino, The Leavitt path algebras of arbitrary graphs, Houston J. Math. (To appear.)

4. G. Abrams, G. Aranda Pino, M. Siles Molina, Finite-dimensional Leavitt path algebras, J. Pure Appl. Algebra. 209 (3) (2007), 753-762.

5. G. Abrams, G. Aranda Pino, M. Siles Molina, Locally finite Leavitt path algebras, Israel J. Math. (To appear.)

6. P. Ara, E. PARdo, Stable rank of Leavitt path algebras. (Preprint.) ArXiv: math.RA/0609354v1 
7. P. Ara, M.A. Moreno, E. Pardo, Nonstable K-Theory for graph algebras, Algebra Represent. Theory (To appear.) ArXiv: math.RA/0412243v3

8. G. Aranda Pino, D. Martín Barquero, C. Martín González, M. Siles Molina, The socle of a Leavitt path algebra. (Submitted.) ArXiv: math.RA/0701637v1

9. G. Aranda Pino, E. Pardo, M. Siles Molina, Exchange Leavitt path algebras and stable rank, J. Algebra 305 (2) (2006), 912-936.

10. G. Aranda Pino, F. Perera, M. Siles Molina, eds., Graph algebras: bridging the gap between analysis and algebra, ISBN: 978-84-9747-177-0, University of Málaga Press, Málaga, Spain (2007).

11. G. Aranda Pino, M. Siles Molina, The Maximal Graded Left Quotient Algebra of a Graded Algebra, Acta Math. Sin. (Engl. Ser.) 22 (1) (2006), 261-270.

12. K. I. Beidar, W. S. Martindale III, A. V. Mikhalev, Rings with generalized identities, Pure and Applied Math., 196, Marcel Dekker, 1996.

13. K. Deicke, J.H. Hong, W. Szymański, Stable rank for graph algebras. Type I graph algebras and their limits, Indiana Univ. Math. 52 (4) (2003), 963-979.

14. J. Firkins, Locally artinian serial rings, Comm. Alg. 32 (4) (2004), 1255-1264.

15. K. FulLER, On rings whose left modules are direct sums of finitely generated modules, Proc. Amer. Math. Soc. 54 (1976), 39-44.

16. M. Gómez Lozano, M. Siles Molina, Quotient rings and Fountain-Gould left orders by the local approach, Acta Math. Hungar. 97 (2002), 287-301.

17. N. Jacobson, Structure of Rings, Amer. Math. Soc. Colloquium Publications, Amer. Math. Soc., Providence, RI (1956).

18. T. Y. LAM, A First Course in Noncommutative Rings. Graduate texts in Mathematics 131, Springer-Verlag, New York (2001).

19. I. RAeBURN, Graph algebras. CBMS Regional Conference Series in Mathematics, 103, Amer. Math. Soc., Providence, (2005).

20. M. Siles Molina, Algebras of quotients of Leavitt path algebras. (Preprint.)

21. M. TOMForde, Uniqueness theorems and ideal structure for Leavitt path algebras, J. Algebra (to appear). ArXiv: math.OA/0612628v3

Department of Mathematics, University of Colorado, Colorado Springs, CO 80933, U.S.A.

E-mail address: abrams@math.uccs.edu

Centre de Recerca Matemàtica, Apartat 50, E-08193, Bellaterra

(BARCELONA), Spain.

E-mail address: garanda@crm.es

Departament of Matemàtiques, Universitat Autònoma de Barcelona, 08193

Bellaterra (Barcelona), Spain.

E-mail address: perera@uab.mat.cat

Departamento de Álgebra, Geometría y Topología, Universidad de Málaga, 29071 Málaga, Spain.

E-mail address: mercedes@agt.cie.uma.es 